\title{
The increasing role of duplex scanning in the follow-up after endovascular repair of abdominal aortic aneurysms
}

This article was published in the following Dove Press journal:

Journal of Vascular Diagnostics

17 April 2014

Number of times this article has been viewed

\section{Raffaele Pulli \\ Walter Dorigo \\ Leonidas Azas \\ Stefano Matticari \\ Aaron Fargion \\ Carlo Pratesi}

Department of Vascular Surgery, University of Florence, Florence, Italy
Correspondence: Raffaele Pulli Department of Vascular Surgery, University of Florence, Florence, Italy $\mathrm{Tel}+390557947607$

Fax +39055 7947448

Email rpulli@unifi.it
Aim: To analyze the results of a follow-up program after endovascular repair of abdominal aortic aneurysm (EVAR) mainly based on duplex ultrasound (DUS) examinations.

Materials and methods: The postoperative surveillance program changed over the time. In recent years (2007-2012) we are used to performing a DUS and an angio-CT scan within one month after EVAR, a DUS at 6 and 12 months and every 6 months thereafter (Group 2, 498 interventions). Data were compared with our historical series (2000-2006; Group 1, 345 interventions.) Perioperative results were recorded. The long-term results were analysed by Kaplan-Meier curves.

Results: The rates of perioperative mortality were $1.1 \%$ and $1.2 \%$ respectively $(P=0.9)$. The cumulative rates of perioperative complications were $16 \%$ and $6.5 \%$, respectively $(P=0.001)$. The rate of reinterventions at 60 months was significantly higher in group 2 than in group 1 ( $29.1 \%$ and $20.1 \%$ respectively, $P=0.03, \log$ rank 4.6 ). Also the 5 -year rate of endoleak was significantly greater in group 2 than group 1 ( $43.3 \%$ and $34.5 \%$ respectively, $P<0.001, \log$ rank 13.4); however, the rate of endoleaks requiring a redo procedure was lower in group $2(35 / 163$, $21.5 \%)$ than in group $1(51 / 108,47 \% ; P<0.001)$.

Conclusion: Data from our study confirm that a DUS-based follow-up program in patients undergoing EVAR is equally sensitive in identifying endoleaks to the CT scan-based program used in past years.

Keywords: duplex ultrasound, contrast-enhanced, endovascular aneurysm repair

\section{Introduction}

Endovascular abdominal aortic aneurysm repair (EVAR) is a less invasive method of treating abdominal aortic aneurysms (AAA), the effectiveness and safety of which in the short and medium term has been widely demonstrated. ${ }^{1,2}$ However, the risk of graft-related complications occurring at any time during follow-up and the consequent need for reinterventions is substantial. ${ }^{3-5}$ For this reason, a lifelong surveillance program for identifying the occurrence of complications over time and monitoring the effectiveness of sac exclusion is mandatory.

The main clinical trials dealing with EVAR have classically used the computed tomography (CT) angiogram, or angio-CT, scan as the primary imaging method during follow-up. However, this technique has several limitations and contraindications, which along with the significant costs, makes the need for alternative follow-up programs unavoidable. For this reason, in recent years different methods of follow-up have been studied and proposed; among them, duplex ultrasound (DUS) has been increasingly used because it is effective, less invasive, and may possibly reduce cost. 
The aim of this study was to analyze the results of a follow-up program after EVAR mainly based on DUS examinations and to compare them with results obtained by classical surveillance with extensive use of angio-CT scans.

\section{Materials and methods}

In the period ranging from January 2000 to December 2012, 843 elective EVAR procedures were performed at our institution. Data concerning these interventions were prospectively collected in a dedicated institutional database, containing main anatomical, clinical, diagnostic, and technical variables. This database also contains perioperative ( $<30$ days) results and all relevant clinical and diagnostic data collected during follow-up.

The postoperative surveillance program changed over the years; in the first period (2000-2006) we performed a plain X-ray of the abdomen at discharge, a DUS at 1, 6, and 12 months and every 6 months thereafter, and an angio-CT scan at 1 month, 12 months, and yearly thereafter (Group 1, 345 interventions). In recent years (2007-2012) we performed a DUS and an angio-CT scan within 1 month after EVAR, a DUS at 6 and 12 months, and every 6 months thereafter, reserving CT scan only in selected patients (Group 2, 498 interventions).

The criteria for performing angio-CT scan after DUS are the suspicion of a type I endoleak, the presence of a type II endoleak associated with sac enlargement, the presence of sac enlargement without any detectable endoleak, the presence of stenosis or occlusion of the endograft, and the evidence of progression of aneurysmal disease above or below the endograft. Moreover, we perform a CT-scan in the presence of any inconclusive DUS due to technical or anatomical problems. All DUS examinations were performed by vascular surgeons experienced in diagnostic ultrasound, with a standardized method following the guidelines of the Italian Society for Vascular Investigations. ${ }^{6}$ The angio-CT was performed with equipment allowing high-resolution, three-dimensional reconstructions in sagittal and coronal orthogonal planes.

Perioperative results were collected in terms of mortality, need for conversion to open repair, access-related and systemic complications (defined as any condition requiring a local surgical treatment or prolonging the hospital stay) and were compared in the two groups with $\chi^{2}$ test.

The long-term results were analyzed by Kaplan-Meier curves in terms of mortality, reintervention (defined as any open or endovascular reintervention involving the endograft), rupture, conversion to open repair, and detection of any endoleak and were compared by the log-rank test. Statistical analysis was performed by means of SPSS version 20.0 for Windows (IBM Corporation, Armonk, NY, USA).

\section{Results}

Demographic data, comorbidities, and risk factors for aneurysmal disease in the two groups are reported in Table 1. Also, the anatomical characteristics of aortic lesions were significantly different in the two groups: patients in group 2 had larger aneurysms, with shorter and more angulated proximal necks than patients in group 1 (Table 2). The endoprostheses used during the two different periods are listed in Table 3. A complete percutaneous access was used in three patients in group 1 and in 115 patients in group 2; 22 patients in group 2 had a unilateral percutaneous access while contralateral femoral artery was approached with a surgical incision. All the remaining patients had a bilateral surgical access.

Two technical failures occurred, one in both groups, leading to immediate conversion to open repair. At the end of the procedure, four type I proximal endoleaks (one in group 1 and three in group 2) were detected, and they were immediately treated with proximal extensions while in 192 cases ( 23 in group 1 and 169 in group 2), a type II endoleak was present and was left untreated. There were ten perioperative deaths,

Table I Demographic data, comorbidities, and risk factors for aneurysmal disease in the two groups

\begin{tabular}{|c|c|c|c|}
\hline & $\begin{array}{l}\text { Group I } \\
\text { (345 interventions) } \\
\text { n (\%) }\end{array}$ & $\begin{array}{l}\text { Group } 2 \\
\text { (498 interventions) } \\
\text { n (\%) }\end{array}$ & P-value \\
\hline Female sex & 17 (5\%) & $69(14 \%)$ & $<0.001$ \\
\hline Age $>79$ years & $73(21 \%)$ & $146(29 \%)$ & 0.004 \\
\hline Arterial hypertension & $223(65 \%)$ & $385(77 \%)$ & $<0.001$ \\
\hline Hyperlipidemia & $133(38 \%)$ & 235 (47\%) & 0.01 \\
\hline Diabetes & 39 (1 1\%) & 58 (I I\%) & 0.9 \\
\hline Coronary artery disease & $163(47 \%)$ & $219(44 \%)$ & 0.3 \\
\hline Chronic obstructive pulmonary disease & $297(86 \%)$ & $397(80 \%)$ & 0.07 \\
\hline Peripheral arterial obstructive disease & $79(23 \%)$ & $89(18 \%)$ & 0.07 \\
\hline Chronic renal failure & $25(7 \%)$ & $53(10.5 \%)$ & 0.1 \\
\hline
\end{tabular}


Table 2 Anatomical characteristics of the abdominal aortic aneurysms

\begin{tabular}{llll}
\hline $\begin{array}{l}\text { Anatomical } \\
\text { features } \\
\text { (mean values) }\end{array}$ & $\begin{array}{l}\text { Group I } \\
\text { (345 interventions) }\end{array}$ & $\begin{array}{l}\text { Group 2 } \\
\text { (498 interventions) }\end{array}$ & P-value \\
\hline $\begin{array}{l}\text { Aneurysm } \\
\text { diameter }\end{array}$ & $51.9 \mathrm{~mm}$ & $54.1 \mathrm{~mm}$ & 0.005 \\
$\begin{array}{l}\text { Length of neck } \\
\text { Neck diameter }\end{array}$ & $25.4 \mathrm{~mm}$ & $23.5 \mathrm{~mm}$ & 0.04 \\
Neck angulation & $15.2^{\circ}$ & $23.2 \mathrm{~mm}$ & $\mathrm{~ns}$ \\
lliac angulation & $38.7^{\circ}$ & $31.3^{\circ}$ & $<0.00$ I \\
lliac diameter & 17.4 & $44.1^{\circ}$ & $\mathrm{ns}$ \\
\hline
\end{tabular}

Abbreviation: ns, not statistically significant.

four in group 1 and six in group 2; the rates of perioperative mortality were $1.1 \%$ and $1.2 \%$, respectively $(P=0.9)$. Local complications occurred in 27 cases in group $1(8 \%)$ and in 14 cases in group $2(3 \%, P=0.001)$, and systemic complications occurred in 27 cases in group $1(8 \%)$ and in 17 cases in group $2(3.5 \%, P=0.005)$; the cumulative rates of perioperative complications were $16 \%$ and $6.5 \%$, respectively $(P=0.001)$.

\section{Follow-up}

The mean duration of follow-up was 39 months (range 1-156 months); in group 1 this value was 64 months (standard deviation [SD] 38.1) while in group 2 it was 21 months (SD 18.1). There were 181 deaths during the follow-up period, eleven ruptures, 19 conversions to open surgery, 124 reinterventions, and 271 endoleaks, with 86 requiring a reintervention. The overall rates of survival, freedom from reinterventions, from rupture, from conversion, and of absence of endoleaks at 10 years were $39.5 \%$ (standard error of the mean [SE] 0.05 ), $59.5 \%$ (SE 0.05), 96.5\% (SE 0.01), 93.1\% (SE 0.02), and $54.5 \%$ (SE 0.03 ), respectively. The events recorded during follow-up in both groups are reported in Table 4. Due to the different durations of follow-up in the two groups, the comparison was performed at 5 years.

The estimated survival at 60 months was $69 \%$ (SE 0.03 ) in group 1 and $73.9 \%$ (SE 0.04) in group $2(P=0.6, \log$-rank 0.6; Figure 1). At the same time interval, there were no significant

Table 3 Endografts used in the two groups

\begin{tabular}{lll}
\hline Endoprosthesis & $\begin{array}{l}\text { Group I } \\
\text { (345 interventions) } \\
\mathbf{n ~ ( \% ) ~}\end{array}$ & $\begin{array}{l}\text { Group 2 } \\
\mathbf{( 4 9 8} \text { interventions) } \\
\mathbf{n ~ ( \% ) ~}\end{array}$ \\
\hline Anaconda & $22(6.5 \%)$ & $139(28 \%)$ \\
Endurant & - & $106(21 \%)$ \\
Excluder & $107(31 \%)$ & $77(15 \%)$ \\
Talent & $142(41 \%)$ & $43(8.5 \%)$ \\
Zenith & $53(15 \%)$ & $119(24 \%)$ \\
Others & $21(6.5 \%)$ & $14(3.5 \%)$ \\
\hline
\end{tabular}

Table 4 Clinical events during follow-up in the two groups

\begin{tabular}{lll}
\hline Events & $\begin{array}{l}\text { Group I } \\
\text { (345 interventions) }\end{array}$ & $\begin{array}{l}\text { Group 2 } \\
\text { (498 interventions) }\end{array}$ \\
\hline Rupture & 6 & 5 \\
Conversion & 14 & 5 \\
Reintervention & 69 & 55 \\
Endoleak & 108 & 163 \\
\hline
\end{tabular}

differences in terms of risk of rupture $(2.1 \%$ and $1.2 \%$, respectively, $P=0.9, \log$ rank 0.1 ) and conversion to open surgery $(5.1 \%$ and $4 \%$, respectively, $P=0.1, \log$ rank 1.1$)$. The rate of reinterventions at 60 months was significantly higher in group 2 than in group $1(29.1 \%$ and $20.1 \%$, respectively, $P=0.03, \log$ rank 4.6; Figure 2).

The 5-year rate of endoleak was significantly greater in group 2 than group 1 (43.3\% and $34.5 \%$, respectively, $P<0.001, \log$ rank 13.4, Figure 3); however, the rate of endoleaks requiring a redo procedure was lower in group 2 $(35 / 163,21.5 \%)$ than in group $1(51 / 108,47 \% ; P<0.001)$.

\section{Discussion}

In many vascular centers worldwide, DUS represents, at the moment, the first-level diagnostic method in the follow-up of aortic endoprostheses, as it provides extremely accurate and reliable information about the graft patency, the behavior of the aneurysmal sac and its changes in diameter, and the presence of endoleaks, their origin, and their evolution over time. These advantages in terms of diagnostic accuracy are associated with a significant reduction of the risks due to the use of ionizing radiation and contrast media with classical

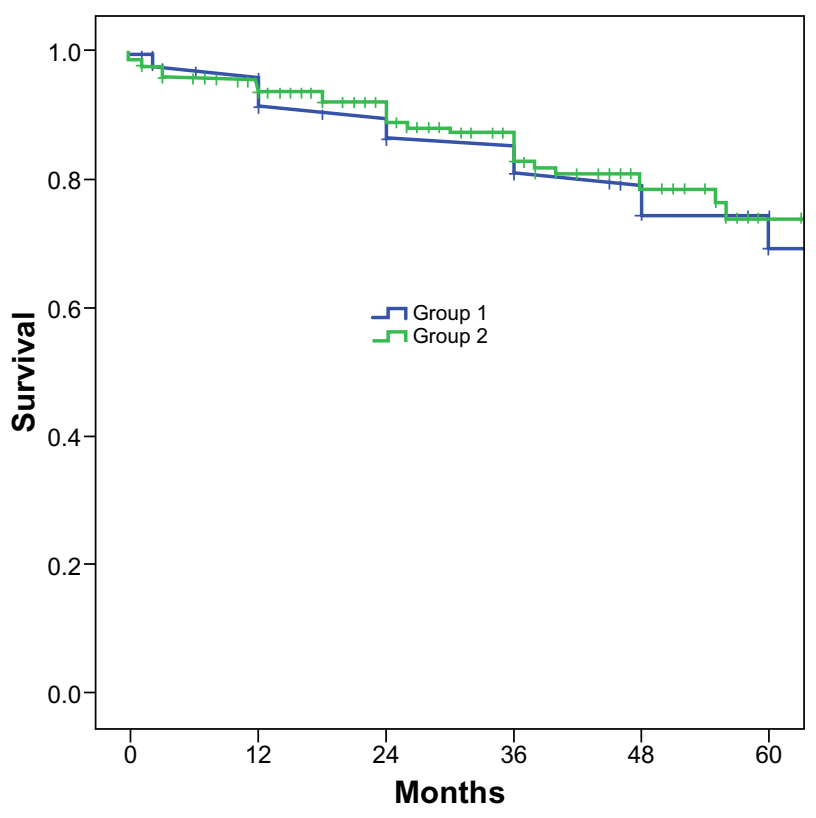

Figure I Kaplan-Meier curve for survival at 60 months in the two groups. 


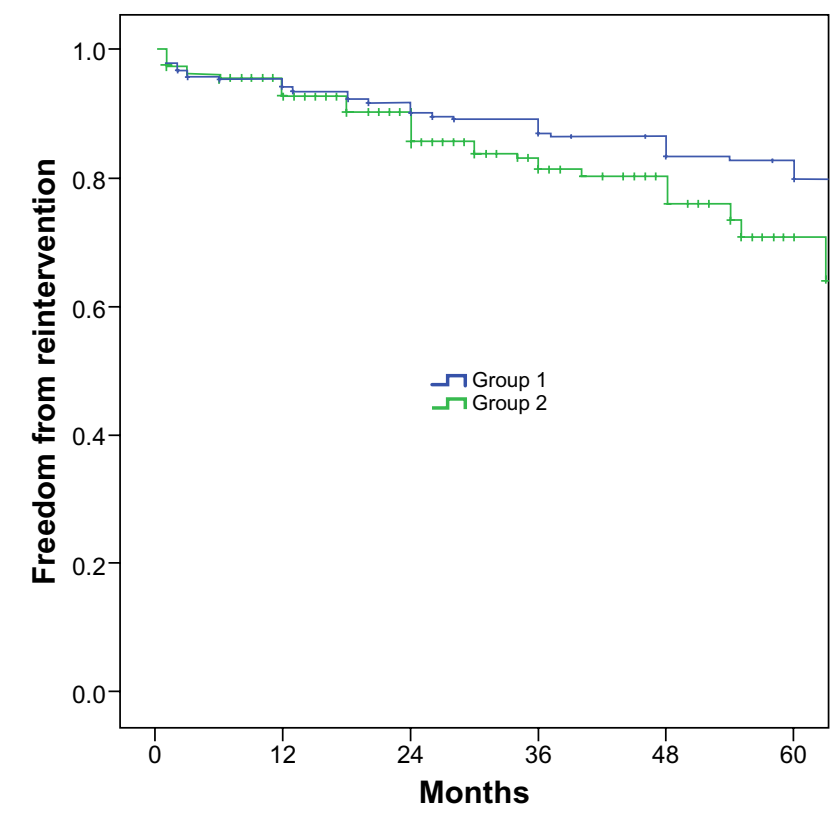

Figure 2 Kaplan-Meier curves for the absence of reinterventions at 60 months in the two groups.

radiological techniques. However, the effectiveness of DUS in identifying endoleaks and in assessing the migration of the graft is still controversial. ${ }^{7}$ In fact, most recent studies confirmed the high reliability of diagnostic ultrasound in terms of specificity and negative predictive power compared to angio-CT, considered the gold standard examination in patients treated with EVAR, but reported conflicting results

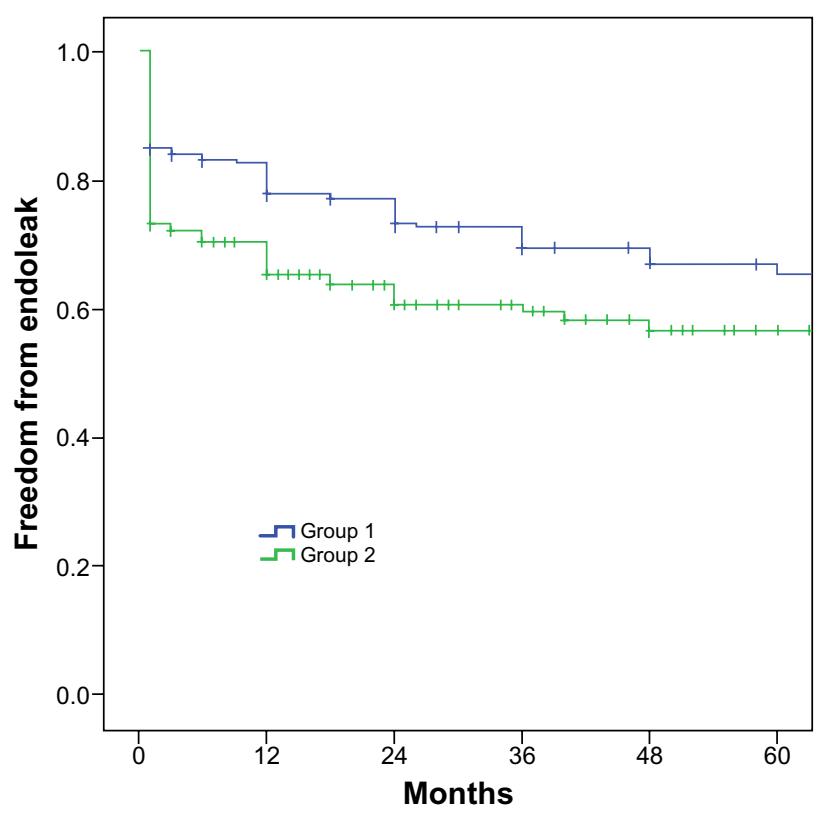

Figure 3 Kaplan-Meier curves for the absence of endoleak at 60 months in the two groups. in terms of sensitivity, with percentages ranging from $43 \%$ to $100 \% .^{10}$

The most recent meta-analysis ${ }^{11}$ confirmed this trend, reporting better values of sensitivity and specificity for angio-CT than DUS. In particular, DUS provides excellent results in the identification of type I and III endoleaks while its efficacy in the detection of type II endoleaks seems to still be poor.

This variability in the sensitivity and positive predictive power between the different studies in the literature is probably related to the intrinsic operator-, patient-, and device-dependent nature of DUS. On the other hand, the differences of surveillance protocols and expertise of the operators in the published studies make the results of metaanalysis difficult to apply to daily clinical practice.

In contrast, several recent monocentric series reported favorable results; Manning et $\mathrm{al}^{12}$ reported in their series a sensitivity value above $70 \%$ for Doppler ultrasound compared to spiral-CT examination (direct examination, arterial and venous phases) and underline how, in the few cases where the Doppler ultrasound lacks the ability to demonstrate the presence of an endoleak, this was always of type II, not associated with increase in the aneurysmal sac. Chaer et $\mathrm{al}^{13}$ reported excellent results in terms of prevention of aneurysmal rupture and conversion to open surgery in their series of 180 patients treated over the past 5 years and followed only by standard DUS, reserving CT scan only for patients with abnormal or suspected findings at DUS. This strategy appears safe (with rates of absence of endoleaks and reintervention more than 96\%) and reliable and reduces the cost of follow-up, which imparts more than $65 \%$ of the total cost of EVAR. In addition, the study is particularly interesting because in some way it changes the traditional parameters for evaluating the effectiveness of a postoperative surveillance program: the ability to identify not all endoleaks, but especially those clinically significantly associated with enlargement of the aneurysmal sac, which represents the main cause of rupture, conversion to open surgery, and reintervention after EVAR. Schmieder et $\mathrm{al}^{14}$ in a recent review of their large series (over 500 procedures), focused exclusively on the detection of endoleaks for which reoperation was necessary and reported a sensitivity (89\%) similar to DUS, significantly better than that achieved by CT scan (58\%). Based on these studies, Doppler ultrasound is used in many centers as the first method of follow-up post-EVAR, reserving CT for particularly complex cases or when DUS is not sufficiently clear. Another possible advantage of DUS is the ability to identify the persistence of pressure within the aneurysmal 
sac in the absence of endoleaks (endotension); in fact, by using the M-mode, the presence of abnormal pulsatility of the aneurysmatic sac may be tested.

Ultrasound techniques, as well as providing satisfactory results in the identification and diagnosis of endoleak, are particularly useful in the follow-up of untreated endoleaks, mainly type II endoleaks not associated with growth of the aneurysmal sac. Recent studies ${ }^{14,15}$ have shown how the hemodynamic characterization at the level of the aneurysmal sac and of the vessels involved in the afferent and efferent blood flow allow to separate the high-speed endoleaks, which rarely tend to seal, from those with lower speed and without outflow vessels, which are supposed to seal spontaneously during follow-up.

The recent introduction and diffusion of ultrasound contrast media seems potentially able to improve the performance of DUS, particularly the ultrasound contrast agents can amplify the intensity of the Doppler signal and improve the identification and visualization of vessels of small size and low speed flows, which often are involved in endoleaks, particularly those of type II.

Initials studies, ${ }^{16,17}$ even though performed on a limited number of patients, had already shown a significant increase in sensitivity and positive predictive power through the use of contrast ultrasound (CEUS) with respect to DUS, with values that could reach and exceed $80 \%$ and $90 \%$, respectively. In particular, the use of ultrasound contrast agents was particularly indicated in the presence of growth of the aneurysmal sac in the absence of endoleaks detected by the usual methods. It was suggested that the pulsatility of the aneurysm sac and its progressive growth are due to the presence of endoleaks at very low flow velocities, not detectable with standard imaging techniques. These data were also confirmed by more recent studies, ${ }^{18}$ which report values of sensitivity and negative predictive value for CEUS similar, if not superior, to those of CT in identifying endoleak. In addition, the use of contrast medium, not only would increase the capacity of DUS to define the presence of an endoleak, but also to identify its origin and then define the type, ${ }^{19}$ thanks to the longer duration of the persistence of contrast within the blood and the possibility of a dynamic angiography evaluation of the morphology and hemodynamics of the leak. In particular, CEUS would seem to allow viewing with extreme accuracy and sensitivity even late endoleaks, ${ }^{20}$ with very low flow velocities and homogeneous distribution of contrast within the thrombus of the aneurysm. Furthermore, after the injection of ultrasound signal amplifiers, not only is the origin of the endoleak highlighted, but the flow velocity and its direction within the sac may be studied.

This type of diagnostic procedure is particularly appropriate in the control of type II endoleaks that, in most cases, do not require further treatment because they undergo spontaneous resolution; however, they must still be checked repeatedly and over time in the short term, until their disappearance. Another potential advantage of the technique is represented by an even better enhancement of the aortic wall, thanks to the passage of ultrasound contrast in the vasa vasorum; this would allow better visualization of the wall to perform more precise measurements of the diameter of the aneurysmal sac compared to only ecographic ${ }^{21}$ examination and similar to those obtained with the CT scan, thereby making more reliable the monitoring of the diameters during follow-up. Based on these findings, the most recent guidelines ${ }^{6}$ indicate how the use of CEUS represents a reliable method in the surveillance program to be used in combination with $\mathrm{CT}$ in the immediate postoperative period and "alone" during the follow-up in all those cases in which no ultrasound reveal complications or investigation is complete and optimal.

The evolution of EVAR and the significant reduction in perioperative complications and hospital stay in comparison with open surgery makes EVAR the treatment of choice in elderly patients at high surgical risk, and it is frequently used in patients at low risk with favorable anatomy.

However, EVAR is not associated with a reduction of the costs, and several studies have shown that the costs of EVAR significantly exceed those of traditional treatment. ${ }^{22-25}$ Moreover, the need for a lifelong program of surveillance and the high reintervention rate during the follow-up contribute to a further increase in the total expense. Recent studies ${ }^{24,25}$ have shown that the postplacement costs of EVAR, both due to reinterventions and to surveillance programs, account for $50 \%$ of the total cost of the procedure. Due to the different costs of the diagnostic techniques used during follow-up, it is clear that a surveillance program including the use of DUS associated with plain X-ray for assessing the integrity of the graft can provide a potential savings of more than $60 \%$ per patient. However, although there are several studies analyzing such a follow-up protocol, there are few data that consider its economic impact. Beeman et al ${ }^{25}$ recently compared in a prospective study the costs of a "standard" surveillance program (DUS and CT scan at 1 and 6 months and then yearly) with those of a program with large use of DUS (DUS and CT scan at 1 month and then only DUS) and showed that the cost of follow-up is halved with the use of ultrasound; similar results have been reported in a retrospective 
study. ${ }^{26}$ However, this substantial savings can be obtained only by reducing mid- and long-term complications; in fact, in the case of complication, CT scan is still required. In this sense it is mandatory to improve the devices and the techniques in order to minimize the risk of endoleaks and the need for additional interventions. Further progress can be achieved with the use of "prophylactic" intraoperative injection of hemostatic glue into the sac or with the use of new generation grafts.

In conclusion, in most studies the controls at 1 month and 1 year are carried out by angio-CT while the intermediate check-up is performed with ultrasound methodology. After the first year of follow-up, the authors agree on running DUS only, reserving the use of radiological methods only in the presence of complications related to the graft. However, many authors still suggest the continued use of CT scan after the first year following surgery because of both little confidence in DUS and to avoid forensic matters. This strategy is expensive and exposes patients to considerable risk, mainly related to the huge amounts of radiation. For this reason, a less aggressive surveillance program has been proposed, performing CT scan only at the first month after surgery and continuing follow-up with the use of ultrasound (DUS and/or CEUS) and plain X-ray of the abdomen to evaluate the position and the structural integrity of the graft, reserving further radiological studies only in the presence of complications. This strategy, although requiring experienced and skilled operators, seems to be cost-effective. Data from our study confirm that a DUS-based follow-up program in patients undergoing EVAR is equally sensitive in identifying endoleaks to the CT scan-based program used in past years. On the other hand, the progressive widening of the indications for EVAR to more complex patients with more complex anatomies has led to an increased rate of complications during follow-up. For these cases as well, DUS surveillance was able to identify complications in patients requiring new treatment.

\section{Disclosure}

The authors report no conflicts of interest in this work.

\section{References}

1. Prinssen M, Verhoeven EL, Buth J, et al; Dutch Randomized Endovascular Aneurysm Management (DREAM) Trial Group. A randomized trial comparing conventional and endovascular repair of abdominal aortic aneurysms. N Engl J Med. 2004;351(16):1607-1618.

2. Greenhalgh RM, Brown LC, Kwong GP, Powell JT, Thompson SG; EVAR trial participants. Comparison of endovascular aneurysm repair with open repair in patients with abdominal aortic aneurysm (EVAR trial 1), 30-day operative mortality results: randomised controlled trial. Lancet. 2004;364(9437):843-848.
3. Blankensteijn JD, de Jong SE, Prinssen M, et al; Dutch Randomized Endovascular Aneurysm Management (DREAM) Trial Group. Two-year outcomes after conventional or endovascular repair of abdominal aortic aneurysms. N Engl J Med. 2005;352(23):2398-2405.

4. EVAR trial participants. Endovascular aneurysm repair versus open repair in patients with abdominal aortic aneurysm (EVAR trial 1): randomised controlled trial. Lancet. 2005;365(9478):2179-2186.

5. EVAR trial participants. Endovascular aneurysm repair and outcome in patients unfit for open repair of abdominal aortic aneurysm (EVAR trial 2): randomised controlled trial. Lancet. 2005;365(9478):2187-2192.

6. Antignani PL, Benedetti-Valentini F, Aluigi L, et al; Italian Society for Vascular Investigation. Diagnosis of vascular diseases. Ultrasound investigations - guidelines. Int Angiol. 2012; 31(5 Suppl 1):1-7.

7. AbuRahma AF, Welch CA, Mullins BB, Dyer B. Computed tomography versus color duplex ultrasound for surveillance of abdominal aortic stent-grafts. J Endovasc Ther. 2005;12(5):568-573.

8. Pages S, Favre JP, Cerisier A, Pyneeandee S, Boissier C, Veyret C. Comparison of color duplex ultrasound and computed tomography scan for surveillance after aortic endografting. Ann Vasc Surg. 2001;15(2): $155-162$.

9. Raman KG, Missig-Carroll N, Richardson T, Muluk SC, Makaroun MS. Color-flow duplex ultrasound scan versus computed tomographic scan in the surveillance of endovascular aneurysm repair. J Vasc Surg. 2003;38(4):645-651.

10. McLafferty RB, McCrary BS, Mattos MA, et al. The use of color-flow duplex scan for the detection of endoleaks. J Vasc Surg. 2002;36(1): $100-104$.

11. Mirza TA, Karthikesalingam A, Jackson D, et al. Duplex ultrasound and contrast-enhanced ultrasound versus computed tomography for the detection of endoleak after EVAR: systematic review and bivariate meta-analysis. Eur J Vasc Endovasc Surg. 2010;39(4): 418-428.

12. Manning BJ, O’Neill SM, Haider SN, Colgan MP, Madhavan P, Moore DJ. Duplex ultrasound in aneurysm surveillance following endovascular aneurysm repair: a comparison with computed tomography aortography. J Vasc Surg. 2009;49(1):60-65.

13. Chaer RA, Gushchin A, Rhee R, et al. Duplex ultrasound as the sole long-term surveillance method post-endovascular aneurysm repair: a safe alternative for stable aneurysms. J Vasc Surg. 2009;49(4): 845-849; discussion 849-850.

14. Schmieder GC, Stout CL, Stokes GK, Parent FN, Panneton JM. Endoleak after endovascular aneurysm repair: duplex ultrasound imaging is better than computed tomography at determining the need for intervention. J Vasc Surg. 2009;50(5):1012-1017; discussion $1017-1018$.

15. Arko FR, Filis KA, Siedel SA, et al. Intrasac flow velocities predict sealing of type II endoleaks after endovascular abdominal aortic aneurysm repair. J Vasc Surg. 2003;37(1):8-15.

16. Giannoni MF, Palombo G, Sbarigia E, Speziale F, Zaccaria A, Fiorani P. Contrast-enhanced ultrasound imaging for aortic stent-graft surveillance. J Endovasc Ther. 2003;10(2):208-217.

17. Napoli V, Bargellini I, Sardella SG, et al. Abdominal aortic aneurysm: contrast-enhanced US for missed endoleaks after endoluminal repair. Radiology. 2004;233(1):217-225.

18. Iezzi R, Basilico R, Giancristofaro D, Pascali D, Cotroneo AR, Storto ML. Contrast-enhanced ultrasound versus color duplex ultrasound imaging in the follow-up of patients after endovascular abdominal aortic aneurysm repair. J Vasc Surg. 2009;49(3):552-560.

19. Carrafiello G, Laganà D, Recaldini C, et al. Comparison of contrastenhanced ultrasound and computed tomography in classifying endoleaks after endovascular treatment of abdominal aorta aneurysms: preliminary experience. Cardiovasc Intervent Radiol. 2006;29(6): 969-974.

20. Bargellini I, Napoli V, Petruzzi P, et al. Type II lumbar endoleaks: hemodynamic differentiation by contrast-enhanced ultrasound scanning and influence on aneurysm enlargement after endovascular aneurysm repair. J Vasc Surg. 2005;41(1):10-18. 
21. Dorigo W, Barbanti E, Azas L, et al. Il follow-up dopo EVAR: come e quando. In Pratesi C, Pulli R, editors. Update in Chirurgia Vascolare. Edizioni Minerva Medica, Torino; 2007:178-188.

22. Dryjski M, O’Brien-Irr MS, Hassett J. Hospital costs for endovascular and open repair of abdominal aortic aneurysm. J Am Coll Surg. 2003;197(1):64-70.

23. Angle N, Dorafshar AH, Moore WS, et al. Open versus endovascular repair of abdominal aortic aneurysms: what does each really cost? Ann Vasc Surg. 2004;18(5):612-618.

24. Noll RE, Tonnessen BH, Mannava K, Money SR, Sternbergh WC. Long-term postplacement cost after endovascular aneurysm repair. J Vasc Surg. 2007;46(1):9-15; discussion 15.
25. Beeman BR, Doctor LM, Doerr K, McAfee-Bennett S, Dougherty MJ, Calligaro KD. Duplex ultrasound imaging alone is sufficient for midterm endovascular aneurysm repair surveillance: a cost analysis study and prospective comparison with computed tomography scan. J Vasc Surg. 2009;50(5):1019-1024.

26. Bendick PJ, Zelenock GB, Bove PG, Long GW, Shanley CJ, Brown OW. Duplex ultrasound imaging with an ultrasound contrast agent: the economic alternative to CT angiography for aortic stent graft surveillance. Vasc Endovascular Surg. 2003;37(3):165-170.

\section{Publish your work in this journal}

Journal of Vascular Diagnostics is an international, peer-reviewed journal of diagnostics, focusing on non invasive vascular investigation methods involved in the evaluation of vascular diseases. The journal is committed to the rapid publication in the fields of vascular diseases. Original research, review, case reports, expert opinion and commentaries

\section{Dovepress}

are all considered for publication. The manuscript management system is completely online and includes a very quick and fair peer-review system, which is all easy to use. Visit http://www.dovepress.com/testimonials.php to read real quotes from published authors.

Submit your manuscript here: http://www.dovepress.com/journal-of-vascular-diagnostics-journal 